\title{
Analytical study of the stability of a two-ball automatic balancer
}

\author{
Chung-Jen Lu*, Ming-Cheng Wang, Shih-Hsuan Huang \\ Department of Mechanical Engineering, National Taiwan University, No. 1, Roosevelt Road, Section 4, Taipei 10617, Taiwan, ROC
}

\section{A R T I C L E I N F O}

\section{Article history:}

Received 22 August 2007

Received in revised form

23 June 2008

Accepted 26 June 2008

\section{Keywords:}

Automatic balancer

Stability

Vibration

Optical disk

\begin{abstract}
A B S T R A C T
A ball-type automatic balancer, which consists of several balls moving on a circular orbit, can effectively reduce the imbalance vibrations of an optical-disk drive. Under proper working conditions, the balls can counter-balance the imbalance of a disk by positioning the appropriate angles relative to the mass center of the disk. These particular positions are referred to as the perfect balancing positions. The proper working conditions depend on the stability of the perfect balancing positions. Much work has been done to determine the stability of the equilibrium positions numerically for some specific values of the system parameters. This paper, in contrast, analytically studies the stability characteristics of the equilibrium positions under several conditions. A theoretical model of an optical-disk drive packed with a two-ball automatic balancer is constructed first. The governing equations of the theoretical model are derived using Lagrange's equations. The closed-form formulae and the existence conditions for the equilibrium positions are presented. Experiments are conducted to verify the theoretical model. The stability of each equilibrium position is determined by Routh's criterion. Finally, general guidelines on the stability of the equilibrium positions are proposed.
\end{abstract}

(c) 2008 Elsevier Ltd. All rights reserved.

\section{Introduction}

The eccentric mass of an optical disk can cause serious vibrations at high rotating speeds. An effective way to reduce the imbalance vibrations is to install a ball-type automatic balancer to the optical-disk drive. A ball-type automatic balancer consists of several balls moving on a circular orbit (Fig. 1). In order to have a clear picture of the working principle of the automatic balancer, consider an undamped rotor system. Assuming that the total mass of the balls is much less than that of the rotor, which includes the disk and the spindle, the behavior of the system is similar to that of a Jeffcott rotor [1]. Because of imbalance, the geometric center $C$ of the disk whirls with the same rotational speed as the disk. The configurations of the system when the rotational speed is subcritical (less than the natural frequency of the system) and supercritical (above the natural frequency) are depicted in Figs. 2a and b, respectively. Note that the center of mass $G$ tends to stay away from the fixed point $O$ in the subcritical speed range while it approaches $O$ in the supercritical speed range. On the other hand, the balls, which can move freely along the orbit, may tend to stay away from $O$ irrespective of the rotational speed. Furthermore, under proper working conditions, the balls will move to specific equilibrium positions where the imbalance vibrations are totally suppressed (Fig. 2c). These equilibrium positions are called the perfect balancing positions and their stability characteristics dictate the proper working conditions.

\footnotetext{
* Corresponding author. Tel:. +8862 3362704; fax: +882223631755.

E-mail address: cjlu@ntu.edu.tw (C.-J. Lu).
} 


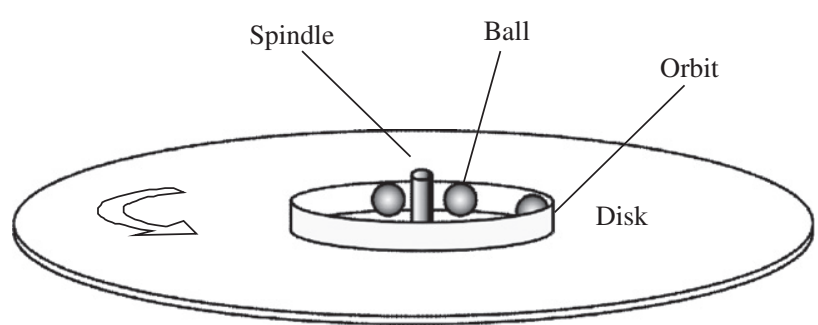

Fig. 1. Schematic diagram of the automatic balancer and rotating disk.

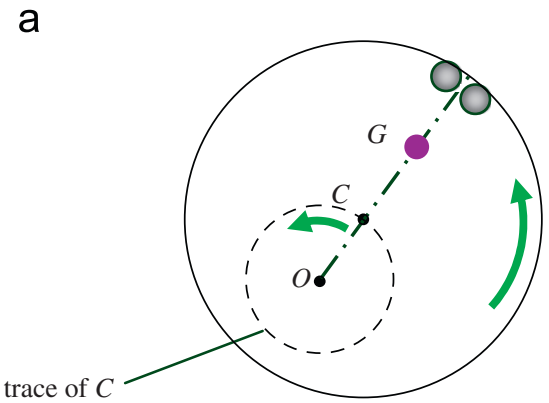

b

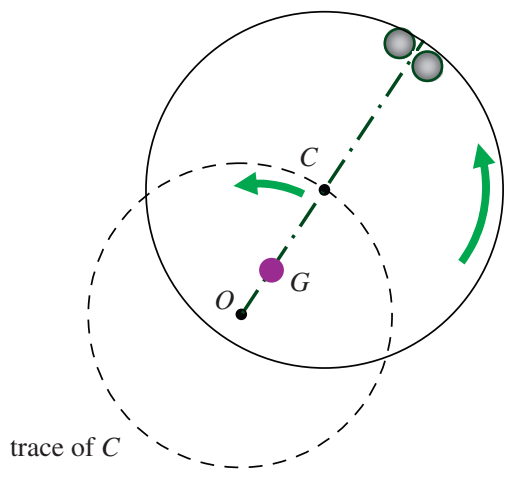

C

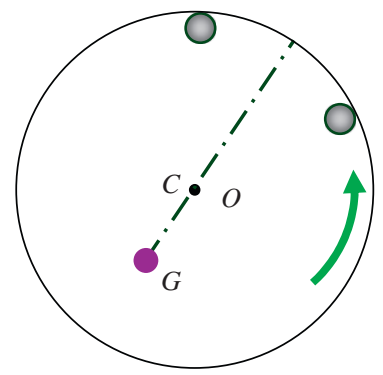

Fig. 2. Schematics of the configurations of the undamped disk-balancer system relative to a fixed reference frame for (a) subcritical speeds, (b) supercritical speeds, and (c) perfect balancing.

Many researchers have studied the properties of the ball-type automatic balancer by either numerical or perturbation methods. Alexander [2] and Cade [3] analyzed the ball-type automatic balancer, but did not describe the working mechanism of the automatic balancer. Bövik and Högfors [4] examined the stability of the perfect balancing positions of a two-ball balancer by the method of multiple scales. Rajalingham et al. [5] investigated the nonlinear system that consists of an undamped rotor and a single-ball automatic balancer. The stability of the equilibrium position was determined by the eigenvalues of the corresponding linearized system. Lee and Moorham [6] numerically determined the stable regions of the perfect balancing positions by the Floquet theory. Hwang and Chung [7] studied the dynamic characteristics of a two-ball balancer with double races. Chung and Ro [8] and Kang et al. [9] determined the stable regions of the perfect balancing positions of a two-ball automatic balancer in the design parameter space. The stability was checked with the variations for a pair of design parameters. Huang et al. [10] studied the dynamic characteristics of a one-ball automatic balancer 
considering the effects of rolling resistance and orbit eccentricity. Chung and Jang [11] considered the effects of the flexibility of the shaft on the performance of the ball-type automatic balancer. The stability of the perfect balancing positions of a two-ball balancer was analyzed by the perturbation method. Kim et al. [12] developed a three-dimensional model and examined the dynamic behavior of a two-ball balancer. Chao et al. [13] evaluated the performance of a two-ball balancer using the method of multiple scales considering the in-plane rotational motions. Rajalingham and Bhat [14] considered the application of a two-ball balancer to a vertical flexible rotor. They pointed out that the system may settle to either a balanced state or a limit cycle. Green et al. [15] conducted a nonlinear bifurcation analysis of a two-ball balancer for eccentric rotors.

These works have indeed furthered our understanding of the dynamic characteristics of the ball-type automatic balancer. However, it is difficult to draw general guidelines on the stability of the equilibrium positions purely based on numerical analyses. Lu [16] analytically studied the stability of the equilibrium positions of a one-ball balancer. He showed that two among the five different types of equilibrium positions are always unstable. Moreover, there is at most one stable equilibrium position at any rotational speed. In this study, we extend Lu's work to a two-ball balancer. The stability of each equilibrium position of a rotating disk equipped with a two-ball automatic balancer is studied analytically. First, the nonlinear governing equations are derived from Lagrange's equations with respect to a co-rotating coordinate system. Then equilibrium positions and the associated linearized equations are obtained. The closed-form formulae and the existence conditions for the equilibrium positions are presented. Experiments are conducted to validate the mathematical model. Finally, the stability of each equilibrium position is determined analytically by Routh's criterion and the results are summarized.

\section{Mathematical model and governing equations}

Fig. 3 depicts schematically the system considered, which consists of a rotating unbalanced disk, an automatic balancer, and the suspension system. The disk with mass $m_{d}$ rotates with a constant angular velocity $\omega$. The mass center $G$ of the disk is located at a distance $e$ from the geometric center $C$. As a result, the imbalance of the disk is $m_{d} e$. The automatic balancer is composed of an annular groove containing two balls and a damping fluid. The balls move freely along the groove and their movements are subject to viscous damping only. Let $d$ denote the radius of the groove, $c_{b}$ the viscous damping constant of the damping fluid, and $m_{b}$ the mass of the ball. The product $m_{b} d$ is the amount of imbalance caused by a single ball and is referred to as the imbalance of a single ball hereafter. The suspension system is isotropic and characterized by equivalent linear springs with stiffness $k$ and viscous dampers with damping constant $c$.

The $x y$-reference frame rotates about a fixed point $O$ with the same speed as the disk. When the disk is at rest, its geometric center $C$ coincides with $O$ at which the supporting springs are undeformed. While rotating, the disk undergoes a translatory motion in this rotating frame and its position is defined by the coordinates $(\hat{x}, \hat{y})$ of the geometric center $C$. The position of the $i$-th ball is given by angle $\beta_{i}$ relative to the mass center $G$ direction. The reason for the choice of this rotating frame is that the equations of motion expressed in this frame are autonomous.

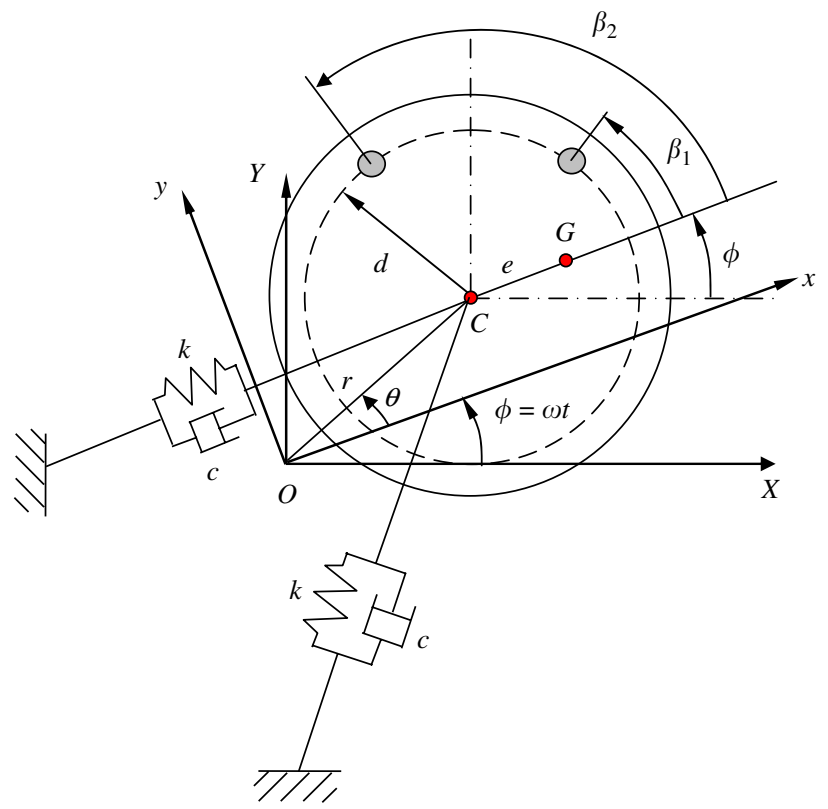

Fig. 3. Schematic diagram of the automatic balancer, rotating disk, and equivalent suspension system. 
The equations of motion are derived from Lagrange's equations given by

$$
\frac{\mathrm{d}}{\mathrm{d} t}\left(\frac{\partial T}{\partial \dot{q}_{k}}\right)-\frac{\partial T}{\partial q_{k}}+\frac{\partial V}{\partial q_{k}}+\frac{\partial R}{\partial \dot{q}_{k}}=0
$$

where $T$ is the kinetic energy, $V$ the potential energy, $R$ the Rayleigh's dissipation function, and $q_{k}$ the generalized coordinates. The kinetic energy can be expressed as

$$
\begin{aligned}
T= & \frac{1}{2} J \omega^{2}+\frac{1}{2} M\left[\dot{\hat{x}}^{2}+\dot{\hat{y}}^{2}+2(\hat{x} \dot{\hat{y}}-\dot{\hat{x}} \hat{y}) \omega+\left(\hat{x}^{2}+\hat{y}^{2}\right) \omega^{2}\right]+\frac{1}{2} m_{d}\left(2 \hat{x} e \omega^{2}+e^{2} \omega^{2}+2 e \dot{\hat{y}} \omega\right) \\
& +\frac{1}{2} m_{b} \sum_{i=1}^{2}\left[d^{2}\left(\dot{\beta}_{i}+\omega\right)^{2}+2 d(\dot{\hat{y}}+\omega \hat{x})\left(\dot{\beta}_{i}+\omega\right) \cos \beta_{i}+2 d(\omega \hat{y}-\dot{\hat{x}})\left(\dot{\beta}_{i}+\omega\right) \sin \beta_{i}\right]
\end{aligned}
$$

where $J$ is the moment of inertia of the equivalent rotor and $M$ the total mass of the system. The potential energy is given by

$$
V=\frac{1}{2} k\left(\hat{x}^{2}+\hat{y}^{2}\right)
$$

Rayleigh's dissipation function can be represented as

$$
R=\frac{1}{2} c\left[\dot{\hat{x}}^{2}+\dot{\hat{y}}^{2}+2(\hat{x} \dot{\hat{y}}-\dot{\hat{x}} \hat{y}) \omega+\left(\hat{x}^{2}+\hat{y}^{2}\right) \omega^{2}\right]+\frac{1}{2} c_{b} d^{2} \sum_{i=1}^{2} \dot{\beta}_{i}^{2}
$$

Substitution of Eqs. (2)-(4) into Eq. (1) yields the nonlinear equations of motion as follows:

$$
\left\{\begin{array}{l}
M \ddot{\hat{x}}-m_{b} d \sum_{i=1}^{2}\left[\left(\dot{\beta}_{i}+\omega\right)^{2} \cos \beta_{i}+\ddot{\beta}_{i} \sin \beta_{i}\right]-m_{d} e \omega^{2}+c \dot{\hat{x}}-2 M \omega \dot{\hat{y}}+\left(k-M \omega^{2}\right) \hat{x}-c \omega \hat{y}=0 \\
M \ddot{\hat{y}}-m_{b} d \sum_{i=1}^{2}\left[\left(\dot{\beta}_{i}+\omega\right)^{2} \sin \beta_{i}-\ddot{\beta}_{i} \cos \beta_{i}\right]+c \dot{\hat{y}}+2 M \omega \dot{\hat{x}}+\left(k-M \omega^{2}\right) \hat{y}+c \omega \hat{x}=0 \\
m_{b} d\left[\left[\ddot{\hat{y}}+2 \omega \hat{\hat{x}}-\omega^{2} \hat{y}\right) \cos \beta_{i}-\left(\ddot{\hat{x}}-2 \omega \dot{\hat{y}}-\omega^{2} \hat{x}\right) \sin \beta_{i}+d \ddot{\beta}_{i}\right]+c_{b} d^{2} \dot{\beta}_{i}=0 \quad i=1,2
\end{array}\right.
$$

In order to simplify the subsequent analysis, the following dimensionless variables are introduced:

$$
\begin{aligned}
& x=\frac{\hat{x}}{d}, \quad y=\frac{\hat{y}}{d}, \quad \tau=\omega_{n} t \\
& c=2 M \varsigma \omega_{n}, \quad c_{b}=2 m_{b} \varsigma_{b} \omega_{n}, \quad \omega_{n}=\sqrt{\frac{k}{M}} \\
& \Omega=\frac{\omega}{\omega_{n}}, \quad \mu_{b}=\frac{m_{b}}{M}, \quad \eta=\frac{m_{b} d}{m_{d} e}
\end{aligned}
$$

It is worth noting that $\Omega$ indicates the ratio of the rotational speed to the natural frequency of the system and $\eta$ the ratio of the imbalance of a single ball to that of the disk. Substituting Eqs. (6) into Eqs. (5) yields the dimensionless equations of motion as follows:

$$
\left\{\begin{array}{l}
x^{\prime \prime}-\mu_{b} \sum_{i=1}^{2}\left[\left(\beta_{i}^{\prime}+\Omega\right)^{2} \cos \beta_{i}+\beta_{i}^{\prime \prime} \sin \beta_{i}\right]+2 \varsigma x^{\prime}-2 \Omega y^{\prime}+\left(1-\Omega^{2}\right) x-2 \varsigma \Omega y=\frac{\mu_{b} \Omega^{2}}{\eta} \\
y^{\prime \prime}-\mu_{b} \sum_{i=1}^{2}\left[\left(\beta_{i}^{\prime}+\Omega\right)^{2} \sin \beta_{i}-\beta_{i}^{\prime \prime} \cos \beta_{i}\right]+2 \varsigma y^{\prime}+2 \Omega x^{\prime}+\left(1-\Omega^{2}\right) y+2 \varsigma \Omega x=0 \\
\mu_{b}\left[\left(y^{\prime \prime}+2 \Omega x^{\prime}-\Omega^{2} y\right) \cos \beta_{i}-\left(x^{\prime \prime}-2 \Omega y^{\prime}-\Omega^{2} x\right) \sin \beta_{i}+\beta_{i}^{\prime \prime}\right]+2 \varsigma_{b} \mu_{b} \beta_{i}^{\prime}=0, \quad i=1,2
\end{array}\right.
$$

where ( $)^{\prime}$ indicates differentiation with respect to $\tau$. Let $\mathbf{q}=\left[x, y, \beta_{1}, \beta_{2}\right]^{\mathrm{T}}$, Eqs. (7) can be put in the matrix form as

$$
\mathbf{M q ^ { \prime \prime }}+\mathbf{D q ^ { \prime }}+\mathbf{g}+\mathbf{f}=\mathbf{0}
$$

where

$$
\begin{aligned}
\mathbf{M} & =\left[\begin{array}{llll}
1 & 0 & -\mu_{b} \sin \beta_{1} & -\mu_{b} \sin \beta_{2} \\
0 & 1 & \mu_{b} \cos \beta_{1} & \mu_{b} \cos \beta_{2} \\
-\mu_{b} \sin \beta_{1} & \mu_{b} \cos \beta_{1} & \mu_{b} & 0 \\
-\mu_{b} \sin \beta_{2} & \mu_{b} \cos \beta_{2} & 0 & \mu_{b}
\end{array}\right] \\
\mathbf{D} & =\left[\begin{array}{llll}
2 \varsigma & -2 \Omega & -2 \mu_{b} \Omega \cos \beta_{1} & -2 \mu_{b} \Omega \cos \beta_{2} \\
2 \Omega & 2 \varsigma & -2 \mu_{b} \Omega \sin \beta_{1} & -2 \mu_{b} \Omega \sin \beta_{2} \\
2 \mu_{b} \Omega \cos \beta_{1} & 2 \mu_{b} \Omega \sin \beta_{1} & 2 \mu_{b} \varsigma_{b} & 0 \\
2 \mu_{b} \Omega \cos \beta_{2} & 2 \mu_{b} \Omega \sin \beta_{2} & 0 & 2 \mu_{b} \varsigma_{b}
\end{array}\right]
\end{aligned}
$$




$$
\begin{aligned}
\mathbf{g} & =\left[\begin{array}{l}
-\mu_{b} \sum_{i=1}^{2}\left(\beta_{i}^{\prime}\right)^{2} \cos \beta_{i} \\
-\mu_{b} \sum_{i=1}^{2}\left(\beta_{i}^{\prime}\right)^{2} \sin \beta_{i} \\
0 \\
0
\end{array}\right] \\
\mathbf{f} & =\left[\begin{array}{l}
\left(1-\Omega^{2}\right) x-2 \varsigma \Omega y-\mu_{b} \Omega^{2}\left(\cos \beta_{1}+\cos \beta_{2}\right)-\frac{\mu_{b} \Omega^{2}}{\eta} \\
2 \varsigma \Omega x+\left(1-\Omega^{2}\right) y-\mu_{b} \Omega^{2}\left(\sin \beta_{1}+\sin \beta_{2}\right) \\
\mu_{b} \Omega^{2}\left(x \sin \beta_{1}-y \cos \beta_{1}\right) \\
\mu_{b} \Omega^{2}\left(x \sin \beta_{2}-y \cos \beta_{2}\right)
\end{array}\right]
\end{aligned}
$$

\section{Equilibrium positions}

The first step in analyzing a nonlinear system is to identify the equilibrium positions. The equilibrium positions, denoted by $\overline{\mathbf{q}}=\left[\begin{array}{llll}\bar{x}, & \bar{y}, & \bar{\beta}_{1}, & \bar{\beta}_{2}\end{array}\right]^{\mathrm{T}}$, can be deduced from Eqs. (7) by suppressing the time derivatives and solving the resulting algebraic equations

$$
\left\{\begin{array}{l}
\left(1-\Omega^{2}\right) \bar{x}-2 \varsigma \Omega \bar{y}-\mu_{b} \Omega^{2}\left(\cos \bar{\beta}_{1}+\cos \bar{\beta}_{2}\right)-\frac{\mu_{b} \Omega^{2}}{\eta}=0 \\
2 \varsigma \Omega \bar{x}+\left(1-\Omega^{2}\right) \bar{y}-\mu_{b} \Omega^{2}\left(\sin \bar{\beta}_{1}+\sin \bar{\beta}_{2}\right)=0 \\
\mu_{b} \Omega^{2}\left(\bar{x} \sin \bar{\beta}_{i}-\bar{y} \cos \bar{\beta}_{i}\right)=0, \quad i=1,2
\end{array}\right.
$$

To solve Eqs. (9), we introduce the polar coordinates

$$
\bar{x}=\bar{r} \cos \bar{\theta}, \quad \bar{y}=\bar{r} \sin \bar{\theta} .
$$

Here $\bar{r}$ denotes the residual vibration of the rotating disk and $\bar{\theta}$ the angle of $O C$ relative to the $x$-axis (Fig. 3). Then Eqs. (9) can be rewritten as

$$
\left\{\begin{array}{l}
\left(1-\Omega^{2}\right) \bar{r} \cos \bar{\theta}-2 \varsigma \Omega \bar{r} \sin \bar{\theta}-\mu_{b} \Omega^{2}\left(\cos \bar{\beta}_{1}+\cos \bar{\beta}_{2}\right)-\frac{\mu_{b} \Omega^{2}}{\eta}=0 \\
2 \varsigma \Omega \bar{r} \cos \bar{\theta}+\left(1-\Omega^{2}\right) \bar{r} \sin \bar{\theta}-\mu_{b} \Omega^{2}\left(\sin \bar{\beta}_{1}+\sin \bar{\beta}_{2}\right)=0 \\
\mu_{b} \Omega^{2} \bar{r} \sin \left(\bar{\beta}_{i}-\bar{\theta}\right)=0, \quad i=1,2
\end{array}\right.
$$

It is worth noting that $\zeta_{b}$ does not appear in the above equations and hence has no effects on the equilibrium positions. Eqs. (10c) require that $\sin \left(\bar{\beta}_{i}-\bar{\theta}\right)=0$ or $\bar{r}=0$. The equilibrium positions associated with $\sin \left(\bar{\beta}_{i}-\bar{\theta}\right)=0$ and $\bar{r}=0$ are called the unbalanced positions and the perfect balancing positions, respectively. The perfect balancing positions are denoted by $P_{0}$, where the subscript 0 indicates zero residual vibration. If the balls are indistinguishable, the unbalanced solutions can be further divided into three cases: (i) $\bar{\beta}_{1}=\bar{\beta}_{2}=\bar{\theta}$, (ii) $\bar{\beta}_{1}=\bar{\beta}_{2}=\bar{\theta}+\pi$, and (iii) $\bar{\beta}_{1}=\bar{\theta}$ and $\bar{\beta}_{2}=\bar{\theta}+\pi$. Including the perfect balancing position (iv) $\bar{r}=0$, there are totally four different cases of solutions to Eqs. (10). Cases (i) and (ii) are equivalent to a single ball with mass $2 m_{b}$ located at $\bar{\beta}=\bar{\theta}$ and $\bar{\beta}=\bar{\theta}+\pi$, respectively. For simplicity, the equilibrium positions associated with (i) and (ii) are referred to as $P_{S 1}$ and $P_{S 2}$, respectively. In case (iii), since the two balls counter-balance each other, the resulting system is equivalent to that with no balancer. The equilibrium positions associated with case (iii) are denoted by $P_{N O}$. Equilibrium positions associated with these four cases are discussed separately below.

(i) $P_{S 1}\left(\bar{\beta}_{1}=\bar{\beta}_{2}=\bar{\theta}\right)$

Substituting $\bar{\beta}_{1}=\bar{\beta}_{2}=\bar{\theta}$ into Eqs. (10a) and (10b) gives, after some rearrangements,

$$
\left\{\begin{array}{l}
\left(\Omega^{2}-1\right) \bar{r}+2 \mu_{b} \Omega^{2}=-\frac{\mu_{b} \Omega^{2} \cos \bar{\theta}}{\eta} \\
2 \varsigma \Omega \bar{r}=-\frac{\mu_{b} \Omega^{2} \sin \bar{\theta}}{\eta}
\end{array}\right.
$$

By squaring and adding we obtain the two possible equilibrium positions, denoted by $P_{S 1}^{ \pm}$. The corresponding radial vibrations are denoted by $\tilde{r}_{S 1}^{ \pm}$and expressed as

$$
\left.\begin{array}{c}
\bar{r}_{S 1}^{+} \\
\bar{r}_{S 1}^{-}
\end{array}\right\}=\mathbf{R}(-2 \eta p \pm \sqrt{\mathbf{D}})
$$


where

$$
\mathbf{R}=\frac{\Omega^{2} \mu_{b}}{\eta\left(p^{2}+q^{2}\right)} \quad \text { and } \quad \mathbf{D}=p^{2}+q^{2}-4 \eta^{2} q^{2}
$$

in which $p$ and $q$ are functions of $\Omega$ as defined below

$$
p=\Omega^{2}-1 \text { and } q=2 \varsigma \Omega
$$

The corresponding $\bar{\theta}^{\prime} s$ are determined by

$$
\bar{\theta}_{S 1}^{ \pm}=\tan ^{-1} \frac{-q \cdot \bar{r}_{S 1}^{ \pm}}{-p \cdot \bar{r}_{S 1}^{ \pm}-2 \mu_{b} \Omega^{2}}
$$

Note that Eq. (11b) implies that $\sin \bar{\theta} \leqslant 0$ and hence $-\pi<\bar{\theta} \leqslant 0$.

Before proceeding further, we need to derive the conditions under which $\bar{r}$ is real and non-negative. As can be seen from Eq. (12), $\bar{r}$ is real if and only if $\mathbf{D}$ is non-negative. From Eq. (13), it follows that:

$$
\mathbf{D} \geqslant 0 \text { if and only if } \eta \leqslant \eta_{c r}
$$

where

$$
\eta_{c r}=\frac{1}{2} \sqrt{1+\frac{p^{2}}{q^{2}}}=\frac{1}{2} \sqrt{1+\frac{\left(\Omega^{2}-1\right)^{2}}{4 \zeta^{2} \Omega^{2}}}
$$

It is easy to see that $\eta_{c r} \geqslant 1 / 2$ and the minimum value $1 / 2$ is reached at $\Omega=1$. Moreover, $\eta_{c r}$ is monotonically decreasing for $0<\Omega<1$ and monotonically increasing for $1<\Omega$. Assuming that $\bar{r}$ is real, i.e. $\eta \leqslant \eta_{c r}$, we proceed to derive the conditions for non-negative $\bar{r}$. Consider the case $\bar{r}_{S 1}^{+}$first. As can be seen from Eqs. (12) and (14), in the subcritical speed range, $\Omega<1, p$ is negative, which in turn implies that $\bar{r}_{S 1}^{+}$is positive. In the supercritical speed range, $\Omega>1, \bar{r}_{S 1}^{+}$is non-negative if $-2 \eta p+\sqrt{\mathbf{D}} \geqslant 0$, or equivalently $\eta \leqslant 1 / 2$. Similarly, we can derive the conditions under which $\bar{r}_{S 1}^{-}$is non-negative. In summary,

$$
\begin{aligned}
& \bar{r}_{S 1}^{+} \geqslant 0 \text { if }\left\{\begin{array}{l}
\eta \leqslant \eta_{c r} \text { for } 0<\Omega<1 \\
\eta \leqslant \frac{1}{2} \quad \text { for } 1 \leqslant \Omega
\end{array}\right. \\
& \bar{r}_{S 1}^{-} \geqslant 0 \text { if } \frac{1}{2} \leqslant \eta \leqslant \eta_{c r} \text { for } 0<\Omega<1
\end{aligned}
$$

(ii) $P_{S 2}\left(\bar{\beta}_{1}=\bar{\beta}_{2}=\bar{\theta}+\pi\right)$

Substitution of $\bar{\beta}_{1}=\bar{\beta}_{2}=\bar{\theta}+\pi$ into Eqs. (10a) and (10b) yields two possible equilibrium positions, denoted by $P_{S 2}^{ \pm}$. The corresponding amplitudes of radial vibrations are denoted by $\bar{r}_{S 2}^{ \pm}$and expressed as

$$
\left.\begin{array}{l}
\bar{r}_{S 2}^{+} \\
\bar{r}_{S 2}^{-}
\end{array}\right\}=\mathbf{R}(2 \eta p \pm \sqrt{\mathbf{D}})
$$

where $\mathbf{R}$ and $\mathbf{D}$ are defined by Eq. (13). The corresponding $\bar{\theta}^{\prime} s$ are determined by

$$
\bar{\theta}_{S 2}^{ \pm}=\tan ^{-1} \frac{-q \cdot \bar{r}_{S 2}^{ \pm}}{-p \cdot \bar{r}_{S 2}^{ \pm}+2 \mu_{b} \Omega^{2}}
$$

By a similar process as before, we can obtain the conditions under which $\bar{r}_{S 2}^{ \pm}$are non-negative. The results are summarized below

$$
\begin{aligned}
& \bar{r}_{S 2}^{+} \geqslant \text {if } 0\left\{\begin{array}{l}
\eta \leqslant \frac{1}{2} \text { for } 0<\Omega<1 \\
\eta \leqslant \eta_{c r} \text { for } 1 \leqslant \Omega
\end{array}\right. \\
& \bar{r}_{S 2}^{-} \geqslant 0 \text { if } \frac{1}{2} \leqslant \eta \leqslant \eta_{c r} \text { for } 1 \leqslant \Omega
\end{aligned}
$$

(iii) $P_{\mathrm{NO}}\left(\bar{\beta}_{1}=\bar{\theta}, \bar{\beta}_{2}=\bar{\theta}+\pi\right)$

In this case, the two balls are located at the two ends of a diameter of the circular orbit and counter-balance each other. Substituting $\bar{\beta}_{1}=\bar{\theta}$ and $\bar{\beta}_{2}=\bar{\theta}+\pi$ into Eqs. (10a) and (10b) gives, after some rearrangements

$$
\left\{\begin{array}{l}
\left(\Omega^{2}-1\right) \bar{r} \cos \bar{\theta}+2 \varsigma \Omega \bar{r} \sin \bar{\theta}=\frac{-\mu_{b} \Omega^{2}}{\eta} \\
\left(\Omega^{2}-1\right) \bar{r} \sin \bar{\theta}-2 \varsigma \Omega \bar{r} \cos \bar{\theta}=0
\end{array}\right.
$$


By squaring and adding we obtain the associated amplitude of radial vibration $\bar{r}_{N O}$ as

$$
\bar{r}_{N O}=\mathbf{R} \sqrt{p^{2}+q^{2}}
$$

where $\mathbf{R}$ is defined by Eq. (13). The corresponding $\bar{\theta}_{N O}$ is determined by

$$
\bar{\theta}_{N O}=\tan ^{-1} \frac{q}{p}
$$

Unlike $P_{S 1}^{ \pm}$and $P_{S 2}^{ \pm}, P_{N O}$ exists unconditionally.

(iv) $P_{0}(\bar{r}=0)$

In this case, the system is perfectly balanced and has no residual vibration. Substituting $\bar{r}=0$ into Eqs. (10) yields

$$
\left\{\begin{array}{l}
\left(\cos \bar{\beta}_{1}+\cos \bar{\beta}_{2}\right)=-\frac{1}{\eta} \\
\left(\sin \bar{\beta}_{1}+\sin \bar{\beta}_{2}\right)=0
\end{array}\right.
$$

which has the solution

$$
\bar{\beta}_{1}=\cos ^{-1}\left(-\frac{1}{2 \eta}\right) \text { and } \bar{\beta}_{2}=-\bar{\beta}_{1}
$$

The results indicate that, when the system is perfectly balanced, the two balls are located symmetrically on the two sides of a diameter passing the center of mass $G$ of the disk. As can be seen from Eq. (25), $\bar{\beta}_{i}$ exists only if $\eta \geqslant(1 / 2)$. Consequently, $P_{0}$ exists only if $\eta \geqslant(1 / 2)$.

The above analysis indicates that $\eta, \Omega$ and $\zeta$ are major factors affecting the equilibrium positions of the system. The existence conditions of an equilibrium position can be seen clearly by identifying its existence regions in the $\Omega-\eta$ plane. Fig. 4 illustrates the existence regions of the equilibrium positions in the $\Omega-\eta$ plane for $\zeta=0.1$. The $\Omega-\eta$ plane is divided into four regions by the curve $\eta_{c r}$ and the line $\eta=0.5$. Note that the value of $\eta_{c r}$, as defined by Eq. (17), varies with $\zeta$, but the qualitative characteristics of the existence regions remain the same. The region below the line $\eta=0.5$ is denoted as region $\mathrm{I}$. In this region, perfect balancing cannot be achieved and only $P_{S 1}^{+}, P_{S 2}^{+}$, and $P_{N O}$ may exist. The region above $\eta=0.5$ is further divided into three regions by the two branches of $\eta_{c r}$. The region between the two branches of $\eta_{c r}$ is indicated as region III. The regions to the left and right of region III are called regions II and IV, respectively. In region II, only $P_{S 1}^{ \pm}$, $P_{0}$, and $P_{N O}$ may exist; in region III, only $P_{N O}$ and $P_{0}$ may exist; in region IV, only $P_{S 2}^{ \pm}, P_{N O}$, and $P_{0}$ may exist. The equilibrium positions that may exist in each region are listed in the figure. As can be seen from the figure, for a given imbalance ratio $\eta$, at most four equilibrium positions may exist at a fixed rotational speed. The equilibrium positions enclosed by $\square$ are unstable in that region. The stability of the equilibrium positions will be studied, experimentally and analytically, later. Note that region III is divided into two parts by the line $\Omega=1$ to clearly show the stable region of $P_{0}$. For the sake of clearness, the configurations of the system associated with each equilibrium position in different $\Omega-\eta$ regions are also shown in Table 1 .

\section{Experimental study}

Experimental study is performed to investigate the fundamental properties of the ABS and validate the mathematical model. Fig. 5 shows a photograph of the experimental apparatus that consists of an ABS, a clear plastic disk, a DC motor, and

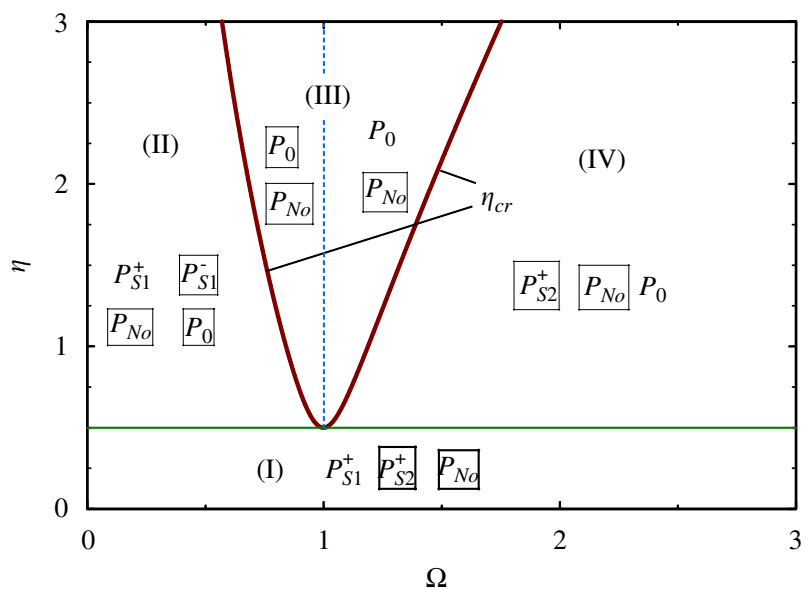

Fig. 4. Existence regions of the equilibrium positions in the $\Omega-\eta$ plane for $\zeta=0.1$. The equilibrium positions enclosed by $\square$ are unstable. 
Table 1

Configurations and existence regions of the equilibrium positions

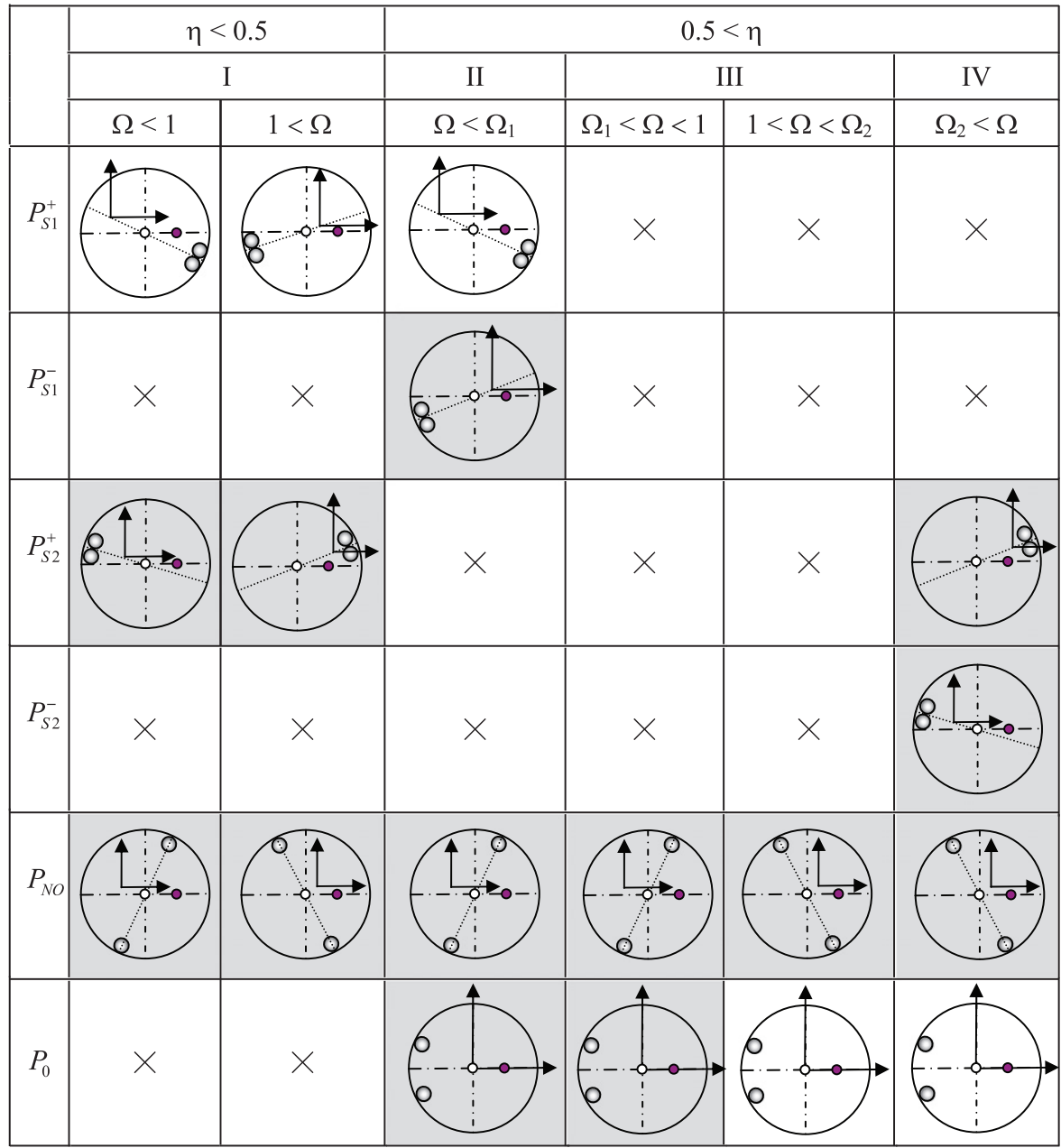

4 - rotating coordinate system.

$\longrightarrow$ - center of mass of the disk.

o-geometric center of the disk.

$\square$ - unstable equilibrium position.

$\Omega_{1.2}$ - solutions of $\eta_{c r}(\Omega)=\eta$ for $\eta>0.5$.

the suspension base. The ABS and the disk were attached firmly to the motor and then mounted on the suspension base. The suspension base was built with four L-shaped beams to realize isotropic stiffness and damping. Modal analysis was applied to determine the natural frequencies of the system. The first two natural frequencies were found to be 25 and $68 \mathrm{~Hz}$. A finite element model of the experimental apparatus was constructed to identify the vibration modes of the system at these two frequencies. The results indicate that the first natural frequency, which is a repeated natural frequency, corresponds to two translational modes in the $x$ - and $y$-directions, respectively. On the other hand, the second natural frequency corresponds to a torsion mode about the spindle. When the system is vibrating at low frequencies where the motion of the system is primarily composed of the two translational modes, the L-shaped beams behave like linear springs directly connected to the geometric center of the disk, which is exactly the case considered in the mathematical model. Therefore, the first measured natural frequency, $25 \mathrm{~Hz}$, is identified as the natural frequency $\omega_{n}$ of the mathematical model and was used to normalize the rotational speed henceforth. The experimental procedure is briefly described below.

The rotor system was balanced prior to experiments. After that, a bolt was locked on the disk at a radial distance $25 \mathrm{~mm}$ to represent the eccentric mass of the disk. Bolts of different sizes were used to adjust the value of the imbalance ratio $\eta$. A laser Doppler vibrometer was used to measure the vibration of one of the L-shaped beams. Video and still cameras were used in conjunction with a strobe light to record the angular positions of the balls. While conducting the experiment, the 


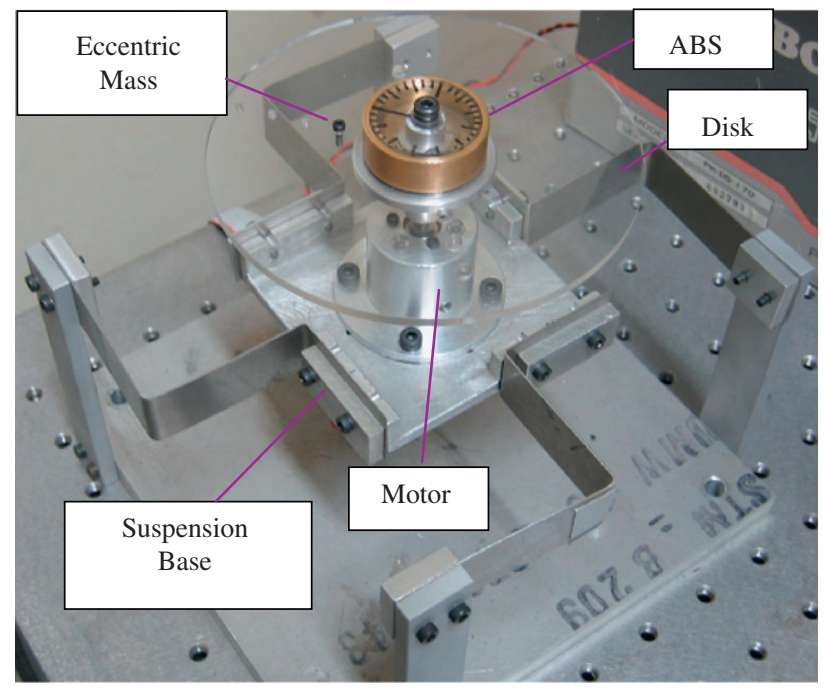

Fig. 5. Photo of the experimental apparatus.

motor was powered to accelerate the disk up to desired speeds. The vibration levels of the L-shaped beam and the ball positions were recorded.

The case $\eta<0.5$ was considered first. Fig. 6(a) shows the levels of steady-state vibration versus rotational speed with and without the ABS for $\eta=0.285$. The vertical dotted lines indicate the first two natural frequencies of the system. No data could be obtained in the vicinity of the natural frequencies due to the large vibration levels. At subcritical rotational speeds, $\Omega<1$, the two balls stick together and stay close to the eccentric mass. Fig. 6 (b) is a typical result showing the positions of the balls at $\Omega=0.84$. In this rotational speed range, the vibration level is increased by the presence of the ABS. On the other hand, at supercritical rotational speeds, $\Omega>1$, the two balls still stick together but stay on the other side of the eccentric mass. Fig. 6(c) shows the positions of the balls at $\Omega=1.56$. In this case, the ABS partially counterbalances the eccentric mass and hence reduces the steady-state vibration level. Table 1 indicates that, for $\eta<0.5$, there exist three equilibrium positions $P_{S 1}^{+}, P_{S 2}^{+}$, and $P_{N 0}$. Comparing Figs. 6(b) and (c) with the configurations of different equilibrium positions shown in Table 1 , one can see that the equilibrium positions observed in the experiment are $P_{S 1}^{+}$. These results imply that, for $\eta<0.5, P_{S 1}^{+}$is stable but $P_{S 2}^{+}$and $P_{N O}$ may be unstable.

Then we examined the case $\eta>0.5$. A larger bolt was used to adjust the value of imbalance ratio $\eta$ to be 0.832 . The associated steady-state vibration levels at different rotational speeds with and without the ABS are shown in Fig. 7(a). At subcritical speeds, the balls stick together and stay close to the eccentric mass, as can be seen in Fig. 7(b). In this case, the ABS increases the vibration levels. On the other hand, in the speed range $1.4<\Omega<2$, the two balls stay on the other side of the eccentric mass and open a suitable angle (Fig. 7(c)) such that the disk is almost completely counterbalanced. As a result, the vibration levels are reduced significantly. Comparison with the equilibrium configurations shown in Table 1 indicates that the equilibrium positions observed in the experiment are $P_{S 1}^{+}$and $P_{0}$ for subcritical and supercritical speeds, respectively. These results imply that $P_{0}$ is stable in some supercritical speed range but unstable for subcritical speeds. The stability of the equilibrium positions is studied analytically in the next section.

\section{Stability analysis}

For an autonomous system, the stability of an equilibrium position can be determined by the eigenvalues of the associated linearized equations. The eigenvalues are roots of the corresponding characteristic polynomial as follows:

$$
F(s)=a_{0} s^{n}+a_{1} s^{n-1}+a_{2} s^{n-2}+\cdots+a_{n-1} s+a_{n}
$$

where the coefficients $a_{i}$, which are functions of the equilibrium position and system parameters, are given explicitly in the Appendix. According to Routh's criterion, information about the stability of the equilibrium position can be obtained directly from the coefficients of the characteristic polynomial [17]. A necessary condition for the equilibrium position to be stable is that all the coefficients of the characteristic polynomial are of the same sign. As can be seen from Eqs. (A.1) and (A.2) in Appendix, both $a_{0}$ and $a_{1}$ are positive. Consequently, the equilibrium position is unstable if any of the coefficients 
a

$\Omega$

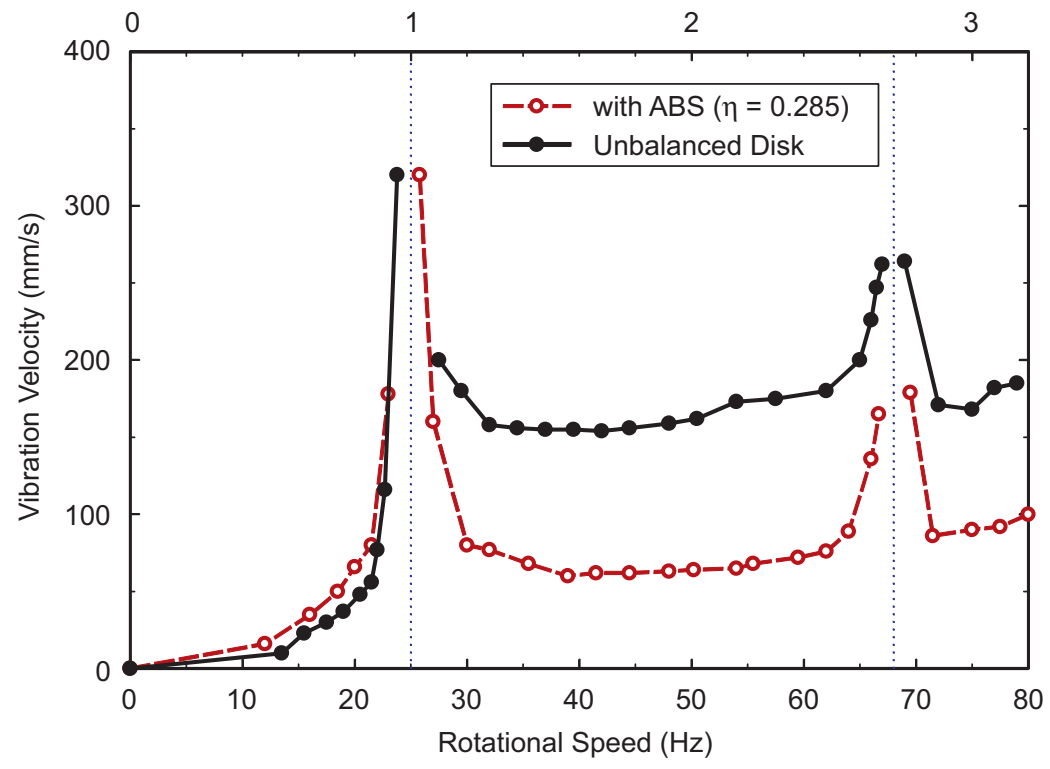

b

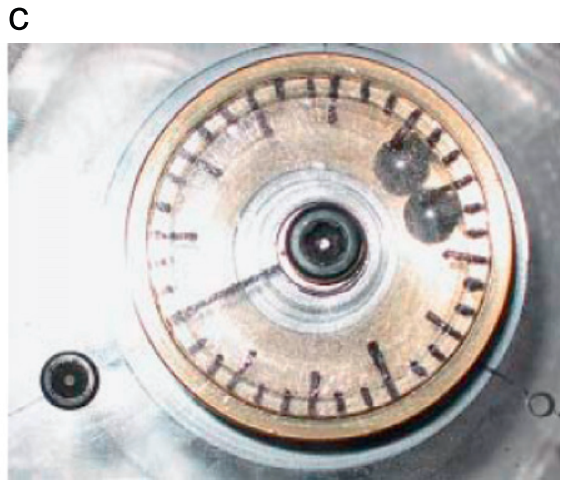

Fig. 6. Effects of ABS for $\eta=0.285$ : (a) vibration spectra with and without ABS; positions of the balls at (b) $\Omega=0.84$ and (c) $\Omega=1.56$.

from $a_{2}$ to $a_{8}$ is negative. This criterion is used in the following paragraphs to analytically show that some equilibrium positions are unstable under certain conditions. The results are listed below.

(S1) The equilibrium position $P_{S 1}^{-}$is unstable.

Substitution of $\bar{\beta}_{1}=\bar{\beta}_{2}=\bar{\theta}$ and $\bar{r}_{S 1}^{-}$(as defined by Eq. (12)) for $\bar{r}$ into Eq. (A.9) gives

$$
a_{8}=-\left(\frac{\mu_{b} \Omega^{6} \sqrt{\mathbf{D}}}{\eta}\right) \bar{r}_{s 1}^{-}<0
$$

Therefore $P_{S 1}^{-}$is unstable.

(S2) The equilibrium position $P_{S 2}^{-}$is unstable.

Substitution of $\bar{\beta}_{1}=\bar{\beta}_{2}=\bar{\theta}+\pi$ and $\bar{r}_{S 2}^{-}$(as defined by Eq. (20)) for $\bar{r}$ into Eq. (A.9) gives

$$
a_{8}=-\left(\frac{\mu_{b} \Omega^{6} \sqrt{\mathbf{D}}}{\eta}\right) \bar{r}_{s 2}^{-}<0
$$

Therefore $P_{S 2}^{-}$is unstable.

(S3) The equilibrium position $P_{S 2}^{+}$is unstable.

In this case, the two balls are located at the same position $\bar{\beta}_{1}=\bar{\beta}_{2}=\bar{\theta}+\pi$. As a result, this particular equilibrium position of the two-ball system is the same as that of a one-ball system with a ball of mass $2 m_{b}$ located at $\bar{\beta}=\bar{\theta}+\pi$. In addition, if the two balls are constrained to move together, then the dynamic characteristics of the two-ball system will be exactly the same as that of the one-ball system. Since this type of equilibrium position for a one-ball system 
a

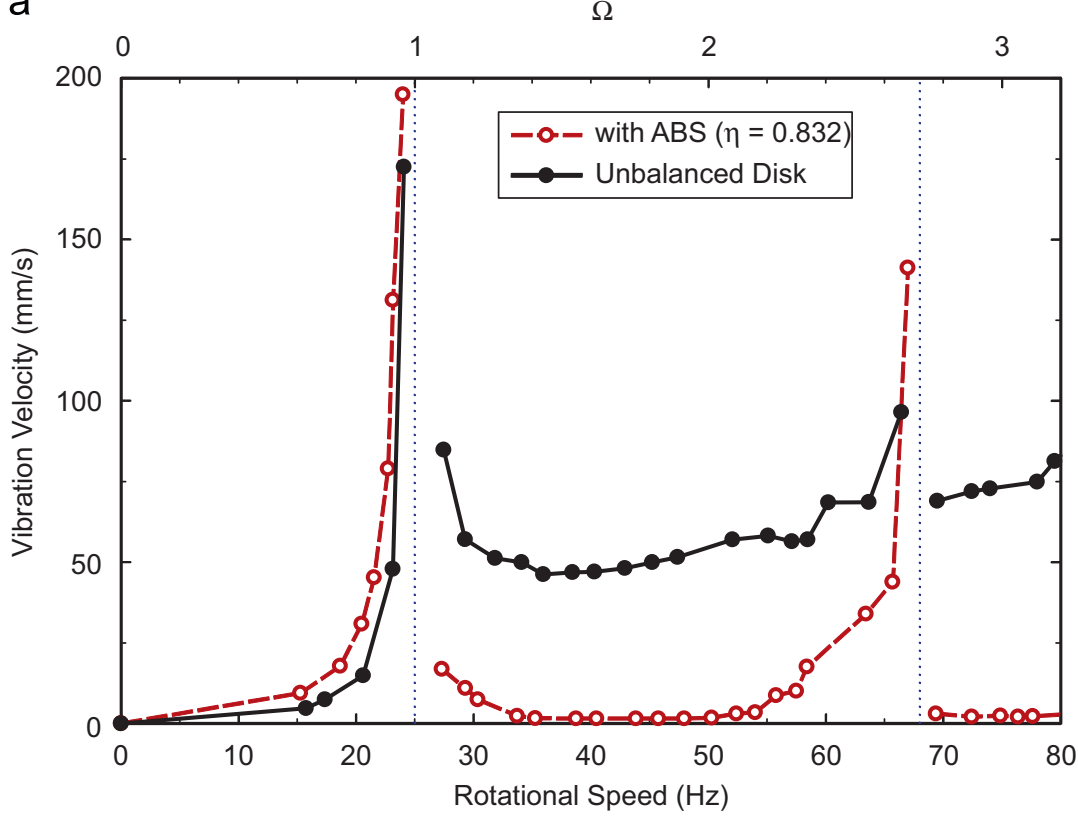

b

C
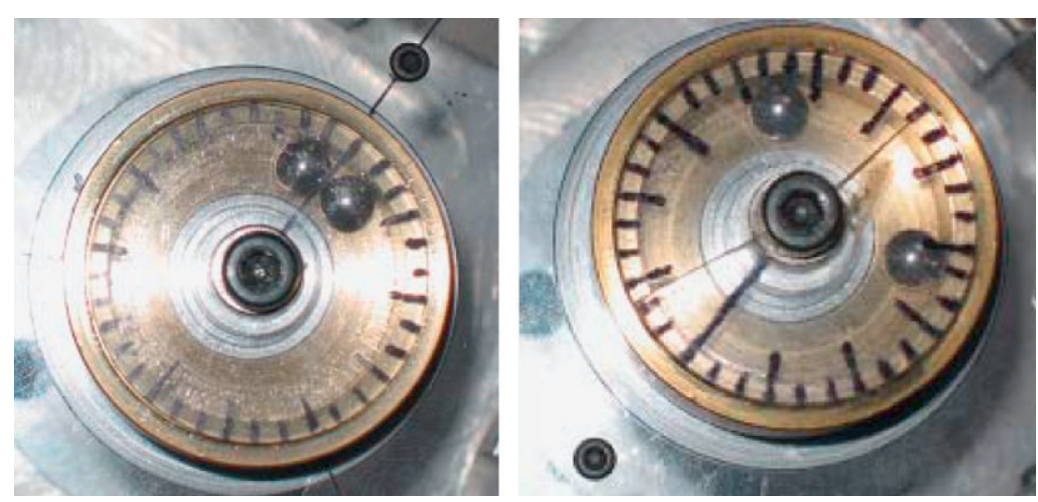

Fig. 7. Effects of ABS for $\eta=0.832$ : (a) vibration spectra with and without ABS; positions of the balls at (b) $\Omega=0.8$ and (c) $\Omega=1.56$.

has been shown to be unstable [16], we can conclude that, for a two-ball system, the equilibrium position $P_{S 2}^{+}$is unstable.

(S4) The equilibrium position $P_{\mathrm{NO}}$ is unstable.

Substituting of $\bar{\beta}_{1}=\bar{\theta}, \bar{\beta}_{2}=\bar{\theta}+\pi$ and $\bar{r}_{N O}$ for $\bar{r}$ into Eq. (A.9) gives $a_{8}=-\bar{r}_{N O}^{2} \Omega^{4}\left(p^{2}+q^{2}\right)<0$.

Therefore $P_{N O}$ is unstable.

(S5) The equilibrium position $P_{0}$ is unstable for $\Omega<1$.

Substitution of $\bar{\beta}_{2}=-\bar{\beta}_{1}$ and $\bar{r}=0$ into Eq. (A.8) gives $a_{7}=4 \mu_{b} \varsigma_{b} \Omega^{4}\left(\Omega^{2}-1\right)$.

Since $a_{7}<0$ for $\Omega<1, P_{0}$ is unstable for $\Omega<1$.

In order to clearly show the above results, the unstable equilibrium positions in Fig. 4 are enclosed by $\square$. However, it must be noted that the equilibrium positions that are not enclosed by $\square$ are not necessarily stable in their existence regions. To verify the above analytical results, the stable regions of the equilibrium positions in the $\Omega-\eta$ plane are determined numerically by the eigenvalues of the corresponding linear systems. Fig. 8 shows a typical result for $\zeta=0.1$ and $\zeta_{b}=0.5$. The light gray and dark gray areas are stable regions of $P_{S 1}^{+}$and $P_{0}$, respectively. No stable equilibrium positions exist in the white area. Comparing Figs. 4 and 8, one can see that the stable region of $P_{0}$ is much smaller than its existence region. Specifically, $P_{0}$ is unstable in some areas inside its existence region; the exact size and shape of the stable region of $P_{0}$ depend on the values of the system parameters. As a result, to achieve perfect balancing, one needs to properly set the values of the system parameters such that $P_{0}$ is stable. 


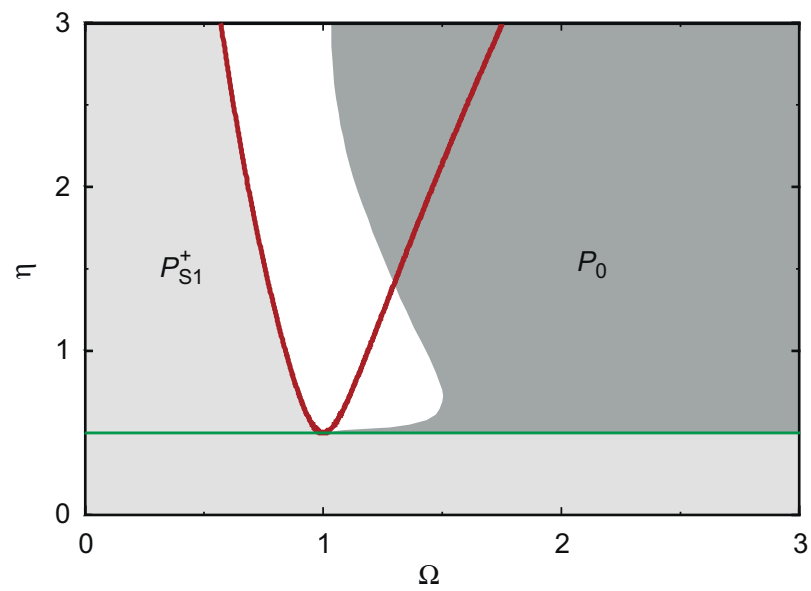

Fig. 8. Stable regions of the equilibrium positions in the $\Omega-\eta$ plane for $\zeta=0.1$ and $\zeta_{b}=0.5$. No stable equilibrium positions exist in the white area.

\section{Conclusion}

Ball-type automatic balancers have been used in the Optical-disk drive industry to suppress rotational vibrations induced by an unbalanced optical disk. Proper conditions under which the imbalance vibrations can be totally suppressed are closely related to the stability of the perfect balancing positions. We have investigated the stability property of a system representative of an optical-disk drive equipped with a two-ball automatic balancer and a suspension system. Experiments were performed to verify the theoretical models. Let $\eta$ denote the ratio of the imbalance of a single ball to that of the disk and $\Omega$ the ratio of the rotational speed to the natural frequency of the system. The existence regions of each equilibrium position in the $\eta-\Omega$ have been identified analytically. General conditions under which some of the equilibrium positions are unstable are proposed. The analytical nature of our investigation has yielded unique understanding of the stability property of the system and insights into the conditions under which perfect balancing can be achieved. The results of the analysis are summarized below.

1. There are totally six different equilibrium positions and four of them are always unstable.

2. There is at most one stable equilibrium position at a rotational speed.

3. The perfect balancing position is unstable for $\Omega<1$.

4. In the areas $\Omega>1$ and $\eta>(1 / 2)$, the only possible stable equilibrium position is the perfect balancing position. The stable region of the perfect balancing position, whose size and shape vary with the values of system parameters, is only a subset of this area.

\section{Acknowledgement}

This work was supported by the National Science Council of R.O.C. under Grant NSC96-2221-E-002-216.

\section{Appendix}

The coefficients of the characteristic polynomial are given below:

$$
\begin{aligned}
a_{0}= & \left(1-2 \mu_{b}\right)+\frac{1}{2} \mu_{b}^{2}\left[1-\cos 2\left(\bar{\beta}_{1}-\bar{\beta}_{2}\right)\right] \\
a_{1}= & 4\left(1-\mu_{b}\right)\left(\varsigma+\varsigma_{b}\right) \\
a_{2}= & \left(1-\mu_{b}\right)\left[2\left(1+\Omega^{2}+4 \varsigma \varsigma_{b}\right)+\bar{r} \Omega^{2}\left(\cos \left(\bar{\beta}_{1}-\bar{\theta}\right)+\cos \left(\bar{\beta}_{2}-\bar{\theta}\right)\right)\right] \\
& +4\left(\varsigma+\varsigma_{b}\right)^{2}+2 \mu_{b}^{2} \Omega^{2}\left[1-\cos 2\left(\bar{\beta}_{1}-\bar{\beta}_{2}\right)\right] \\
a_{3}= & 2\left\{2\left[4 \varsigma^{2} \varsigma_{b}+\varsigma_{b}\left(2-\mu_{b}\right)\left(1+\Omega^{2}\right)+\varsigma\left(1+4 \varsigma_{b}^{2}+\left(1+2 \mu_{b}\right) \Omega^{2}\right)\right]\right. \\
& \left.+\bar{r} \Omega^{2}\left[\varsigma\left(2-\mu_{b}\right)+\varsigma_{b}\right]\left(\cos \left(\bar{\beta}_{1}-\bar{\theta}\right)+\cos \left(\bar{\beta}_{2}-\bar{\theta}\right)\right)\right\}
\end{aligned}
$$




$$
\begin{aligned}
& a_{4}=\left(\Omega^{2}-1\right)^{2}+8 \varsigma_{b}\left[\varsigma_{b}+2 \varsigma\left(1+\varsigma \varsigma_{b}\right)\right]+4\left[\varsigma^{2}+3 \mu_{b}+4 \varsigma \varsigma_{b}\left(1+\mu_{b}\right)+2 \varsigma_{b}^{2}\right] \Omega^{2} \\
& +2 \mu_{b} \Omega^{4}+3 \mu_{b}^{2} \Omega^{4}\left(1-\cos 2\left(\bar{\beta}_{1}-\bar{\beta}_{2}\right)\right)+\bar{r}^{2} \Omega^{4} \cos \left(\bar{\beta}_{1}-\bar{\theta}\right) \cos \left(\bar{\beta}_{2}-\bar{\theta}\right) \\
& +\bar{r} \Omega^{2}\left[4 \varsigma\left(\varsigma+2 \varsigma_{b}\right)+\left(2-\mu_{b}\right)\left(1+\Omega^{2}\right)\right]\left(\cos \left(\bar{\beta}_{1}-\bar{\theta}\right)+\cos \left(\bar{\beta}_{2}-\bar{\theta}\right)\right) \\
& a_{5}=4\left\{\bar{r}^{2} \varsigma \Omega^{4} \cos \left(\bar{\beta}_{1}-\bar{\theta}\right) \cos \left(\bar{\beta}_{2}-\bar{\theta}\right)+\varsigma_{b}\left(\Omega^{2}-1\right)^{2}+4 \varsigma \varsigma_{b}\left(1+\Omega^{2}\right)\right. \\
& +2\left(2 \varsigma^{2}+3 \mu_{b}\right) \varsigma_{b} \Omega^{2}+\mu_{b}\left(3 \varsigma+\varsigma_{b}\right) \Omega^{4}+\bar{r} \Omega^{2}\left[\varsigma+\left(1+2 \varsigma^{2}\right) \varsigma_{b}\right. \\
& \left.\left.+\left(\varsigma+\varsigma \mu_{b}+\varsigma_{b}\right) \Omega^{2}\right]\left(\cos \left(\bar{\beta}_{1}-\bar{\theta}\right)+\cos \left(\bar{\beta}_{2}-\bar{\theta}\right)\right)\right\} \\
& a_{6}=24 \varsigma_{b} \mu_{b} \Omega^{4}+2 \mu_{b} \Omega^{4}\left[\left(\Omega^{2}-1\right)+2 \mu_{b} \Omega^{2} \sin ^{2}\left(\bar{\beta}_{1}-\bar{\beta}_{2}\right)\right]+4 \varsigma_{b}^{2}\left[\left(\Omega^{2}-1\right)^{2}+4 \varsigma^{2} \Omega^{2}\right] \\
& +2 \bar{r}^{2} \Omega^{4}\left(1+\Omega^{2}+2 \varsigma^{2}\right) \cos \left(\bar{\beta}_{1}-\bar{\theta}\right) \cos \left(\bar{\beta}_{2}-\bar{\theta}\right)+\bar{r} \Omega^{2}\left[\left(\Omega^{2}-1\right)^{2}+4 \varsigma^{2} \Omega^{2}\right. \\
& \left.+8 \varsigma \varsigma_{b}\left(1+\Omega^{2}\right)+\mu_{b} \Omega^{2}\left(6+\Omega^{2}\right)\right]\left(\cos \left(\bar{\beta}_{1}-\bar{\theta}\right)+\cos \left(\bar{\beta}_{2}-\bar{\theta}\right)\right) \\
& a_{7}=2 \Omega^{2}\left\{2 \mu_{b} \varsigma_{b} \Omega^{2}\left(\Omega^{2}-1\right)+\bar{r}\left[2 \bar{r} \varsigma \Omega^{2}\left(1+\Omega^{2}\right) \cos \left(\bar{\beta}_{1}-\bar{\theta}\right) \cos \left(\bar{\beta}_{2}-\bar{\theta}\right)\right.\right. \\
& \left.\left.+\left[\varsigma_{b}\left(\Omega^{2}-1\right)^{2}+\varsigma \Omega^{2}\left(4 \varsigma \varsigma_{b}+3 \mu_{b} \Omega^{2}\right)\right]\left(\cos \left(\bar{\beta}_{1}-\bar{\theta}\right)+\cos \left(\bar{\beta}_{2}-\bar{\theta}\right)\right)\right]\right\} \\
& a_{8}=\Omega^{4}\left\{\bar{r}^{2}\left[\left(\Omega^{2}-1\right)^{2}+4 \varsigma^{2} \Omega^{2}\right] \cos \left(\bar{\beta}_{1}-\bar{\theta}\right) \cos \left(\bar{\beta}_{2}-\bar{\theta}\right)\right. \\
& \left.+\mu_{b} \Omega^{2}\left[\bar{r}\left(\Omega^{2}-1\right)\left(\cos \left(\bar{\beta}_{1}-\bar{\theta}\right)+\cos \left(\bar{\beta}_{2}-\bar{\theta}\right)\right)+\mu_{b} \Omega^{2} \sin ^{2}\left(\bar{\beta}_{1}-\bar{\beta}_{2}\right)\right]\right\}
\end{aligned}
$$

\section{References}

[1] L. Meirovitch, Elements of Vibration Analysis, McGraw-Hill, New York, 1986.

[2] J.D. Alexander, An automatic dynamic balancer, in: Proceedings for the Second Southeastern Conference, 1964, pp. 415-426.

[3] J.W. Cade, Self-compensating balancing in rotating mechanism, Design News (1965) 234-239.

[4] P. Bövik, C. Högfors, Autobalancing of rotors, Journal of Sound and Vibration 111 (1986) 429-440.

[5] C. Rajalingham, R.B. Bhat, S. Rakheja, Automatic balancing of flexible vertical rotors using a guided ball, International Journal of Mechanical Sciences $40(1998) 825-834$

[6] J. Lee, W.K.V. Moorham, Analytical and experimental analysis of a self-compensating dynamic balancer in a rotating mechanism, ASME Journal of Dynamic Systems, Measurement and Control 118 (1996) 468-475.

[7] C.-H. Hwang, J. Chung, Dynamic analysis of an automatic ball balancer with double races, JSME International Journal, Series C 42 (1999) $265-272$.

[8] J. Chung, D.S. Ro, Dynamic analysis of an automatic dynamic balancer for rotating mechanisms, Journal of Sound and Vibration 228 (1999) $1035-1056$

[9] J.R. Kang, C.P. Chao, C.L. Huang, C.K. Sung, The dynamics of a ball-type balancer system equipped with a pair of free-moving balancing masses, ASME Journal of Vibration and Acoustics 123 (2001) 456-465.

[10] W.Y. Huang, C.P. Chao, J.R. Kang, C.K. Sung, The application of ball-type balancers for radial vibration reduction of high-speed optic disk drives, Journal of Sound and Vibration 250 (2002) 415-430.

[11] J. Chung, I. Jang, Dynamic response and stability analysis of an automatic ball balancer for a flexible rotor, Journal of Sound and Vibration 259 (2003) $31-43$.

[12] W. Kim, D.-J. Lee, J. Chung, Three-dimensional modelling and dynamic analysis of an automatic ball balancer in an optical disk drive, Journal of Sound and Vibration 285 (2005) 547-569.

[13] P.C.P. Chao, C.-K. Sung, C.-C. Wang, Dynamic analysis of the optical disk drives equipped with an automatic ball balancer with consideration of torsional motions, ASME Journal of Applied Mechanics 72 (2005) 826-842.

[14] C. Rajalingham, R.B. Bhat, Complete balancing of a disk mounted on a vertical cantilever shaft using a two ball automatic balancer, Journal of Sound and Vibration 290 (2006) 169-191.

[15] K. Green, A.R. Champneys, N.J. Lieven, Bifurcation analysis of an automatic dynamic balancing mechanism for eccentric rotors, Journal of Sound and Vibration 291 (2006) 861-881.

[16] C.J. Lu, Stability analysis of a single-ball automatic balancer, ASME Journal of Vibration and Acoustics 128 (2006) 122-125.

[17] K. Ogata, Modern Control Engineering, Prentice-Hall, Englewood Cliffs, NJ, 2001. 